\title{
Modelling cell aggregation using a modified swarm model
}

\author{
F. Georgiou $^{\text {a }}$ (D), N. Thamwattana ${ }^{a}$, and B.P. Lamichhane ${ }^{a}$ \\ ${ }^{a}$ School of Mathematical and Physical Sciences, The University of Newcastle, Callaghan NSW 2203 Australia \\ Email: fillipe.georgiou@uon.edu.au
}

\begin{abstract}
Cell aggregation and sorting are responsible for the formation, stability, and breakdown of tissue. A key mechanism for cell aggregations and sorting is that of cell-cell adhesion, a process by which cells bind or stick to each other through transmembrane proteins. This process is able to achieve cell sorting via the differential adhesion hypothesis (DAH) (Steinberg (1962b a c)). Armstrong et al. (2006) proposed a non-local advection model that was able to simulate the DAH. In their study, cells were modelled using a conservative system acting on cell density. The equations allowed for only two types of movement, random diffusive and directed adhesive movement with the adhesive movement taking into account cells within a finite sensing radius. Using the model with differing cell adhesion values they were able to simulate engulfment, partial engulfment, mixing, and sorting patterns between two cell types in both one and two dimensions.
\end{abstract}

The aggregation of cells can be considered as a type of swarming, in that it is the collective behaviour of a large number of self propelled entities (Loan and Evans (1999)). Examples of macroscopic biological swarms include locust swarms, ungulate herds, fish schools, bird flocks, etc. Non-local swarming models have been used to successfully model these phenomena (see Bernoff and Topaz (2013)). Based on the principle of conservation of mass, a fixed population density moves at a velocity that arises as a result of social interactions (Mogilner and Edelstein-Keshet (1999)), giving rise to an equation of the form

$$
\frac{\partial \rho}{\partial t}+\nabla \cdot(-\nabla(Q(x) * \rho) \rho)=0
$$

with $Q(x)$ being a social potential function used to describe the social interactions between individuals.

In this paper we look at the Armstrong et al. (2006) model of cell-cell adhesion and recreate it by extending the swarm modelling techniques to equations of the form

$$
\frac{\partial \rho}{\partial t}+\nabla \cdot(-\nabla(Q(x) * f(\rho)) \rho)=0
$$

In doing so we find that by modelling in this way we are able to capture the same qualitative behaviour as the original model with a vastly reduced computational cost. We also derive a numerical scheme to simulate the model in one dimension in such a way that it can be easily adapted to other swarm problems. We find that the convergence rate of the numerical scheme is greater than 1.7 in all of the scenarios presented.

Keywords: Cell modelling, numerical methods, swarm modelling, cell-cell adhesion 


\section{INTRODUCTION}

Cell aggregation and sorting are responsible for the formation, stability, and breakdown of tissue. A key mechanism for cell aggregations and sorting is that of cell-cell adhesion, a process by which cells bind or stick to each other through transmembrane proteins. This process is able to achieve cell sorting via the differential adhesion hypothesis (DAH). Based on thermodynamic principles it states that cells will reorganise themselves in order to minimize their interfacial free energy, i.e., they will rearrange in such a way that their intercellular bonds are strongest. When cell populations are thought of as a liquid this results in cell populations with lower surface tension enveloping those with higher surface tensions (Foty and Steinberg (2005)).

Armstrong et al. (2006) created a non-local advection model that was able to simulate the DAH. In their paper, cells were modelled using a conservative system acting on cell density. The equations allowed for only two types of movement, random diffusive and directed adhesive movement. The adhesive movement was found by adding up all the forces acting on a cell within a sensing radius $R$. Using the model with differing cell adhesion values they were able to simulate engulfment, partial engulfment, mixing, and sorting patterns in both one and two dimensions. In Sheratt et al. (2009), generalised cell kinetics (i.e., cell births and death) were added giving rise to the following equations

$$
\begin{aligned}
\frac{\partial u_{i}}{\partial t} & =D_{i} \frac{\partial^{2} u_{i}}{\partial x^{2}}-\frac{\partial}{\partial x}\left(u_{i} K_{i}(\underline{u})\right)+c_{i}(\underline{u}), \\
K_{i}(\underline{u}) & =\frac{\phi_{i}}{R_{i}} \int_{-R_{i}}^{R_{i}} \sum_{j=1}^{N} S_{i j} g_{i j}\left(u_{i}\left(x+x_{0}\right), u_{j}\left(x+x_{0}\right)\right) \omega_{i}\left(x_{0}\right) d x_{0},
\end{aligned}
$$

where $u_{i}(x, t)$ represents the density of cell type $i, \underline{u}$ represents a vector where the $i$ th element is $u_{i}(x, t), D_{i}$ is the diffusion coefficient of cell type $i, c_{i}$ is a function representing cell kinetics, $R_{i}$ is the sensing radius, $\phi_{i}$ is a constant of proportionality related to viscosity, $S_{i j}$ is the adhesion strength between cell types $i$ and $j, g_{i j}\left(u_{i}\left(x+x_{0}\right), u_{j}\left(x+x_{0}\right)\right)$ describes the nature of the forces and their dependence on local cell density, $\omega_{i}\left(x_{0}\right)$ describes how the direction and magnitude of the force changes with $x_{0}$.

The aggregation of cells can be considered as a type of swarming, in that it is the collective behaviour of a large number of self propelled entities (Loan and Evans (1999)). Examples of macroscopic biological swarms include locust swarms, ungulate herds, fish schools, bird flocks, etc. Non-local swarming models have been used to successfully model these phenomena (see Bernoff and Topaz (2013)). Based on the principle of conservation of mass, a fixed population density moves at a velocity that arises as a result of social interactions (Mogilner and Edelstein-Keshet (1999)), giving rise to an equation of the form

$$
\frac{\partial \rho}{\partial t}+\nabla \cdot(-\nabla(Q(x) * \rho) \rho)=0
$$

with $Q(x)$ being a social potential function used to describe the social interactions between individuals.

This paper explores extensions to the swarm modelling of the form

$$
\frac{\partial \rho}{\partial t}+\nabla \cdot(-\nabla(Q(x) * f(\rho)) \rho)=0
$$

to model cell aggregations. We compare the results from the new model against Armstrong et al. (2006) and look at the computational advantages it might present. In Section 2 we derive a model that exhibits similar behaviour to that of Armstrong et al. (2006). Then, in Section 3 we derive a numerical scheme that is easily adapted to other non-local advection models. In Section 4 present at simulations of the cell behaviour and estimate the error and convergence of our numerical scheme.

\section{MODEL}

In a similar fashion to Topaz et al. (2012) we begin with two cell populations $u$ and $v$. We assume that the cells only undergo motion due to random diffusive movement and social interaction forces. However, we model the interaction force using a function of cell density as opposed to simply just the density (i.e. $-\nabla(Q(x) * f(\rho))$ as opposed to $-\nabla(Q(x) * \rho))$. This results in the two equations

$$
\begin{aligned}
& \frac{\partial u}{\partial t}=-\nabla \cdot\left(\mathbf{v}_{u} u\right)+D \frac{\partial^{2} u}{\partial x^{2}}, \\
& \frac{\partial v}{\partial t}=-\nabla \cdot\left(\mathbf{v}_{v} v\right)+D \frac{\partial^{2} v}{\partial x^{2}}
\end{aligned}
$$




\begin{tabular}{|l|l|}
\hline Symbol & Definition \\
\hline$\Delta x$ & spatial distance between grid points in the $x$ direction \\
\hline $\mathbf{x}$ & vector representing the discretised spatial grid \\
\hline$x_{i}$ & $x$ value of the grid point $i$ \\
\hline$U_{i}, V_{i}$ & Approximate function values of $u$ and $v$ at the grid point $i$ \\
\hline $\mathbf{U}, \mathbf{V}$ & vectors representing the discretised functions $u$ and $v$ \\
\hline$v_{u, i}$ & Approximate value of the velocity function $\mathbf{v}_{u}$ at grid point $i$ \\
\hline$v_{v, i}$ & Approximate value of the velocity function $\mathbf{v}_{v}$ at grid point $i$ \\
\hline
\end{tabular}

Table 1. Definitions of symbols used in relation to an arbitrary grid point $i$.

where

$$
\mathbf{v}_{u}=-\nabla\left(Q_{u} * f(u, v)\right) \text { and } \mathbf{v}_{v}=-\nabla\left(Q_{v} * g(u, v)\right) .
$$

To represent the short range cell-cell adhesion based attraction as well as longer ranged attractive forces, we select a social interaction function that gives a strong attraction at close range that rapidly diminishes. A common choice is of the form

$$
Q_{u, v}=e^{-\frac{|x|}{r}}
$$

where $r$ is the length scale that represents typical cell interaction distances.

Finally, we argue that areas of particularly high or low density have fewer free adhesion molecules and provide less avenues for attraction, the former due to the greater presence of bondings and crowding, and in the latter case due simply to less cells being present. This gives a good selection of $f$ and $g$ as

$$
\begin{aligned}
& f(u, v)=\max \left\{\left(S_{u} u+C v\right)(1-u-v), 0\right\}, \\
& g(u, v)=\max \left\{\left(S_{v} v+C u\right)(1-u-v), 0\right\},
\end{aligned}
$$

where $S_{u}$ and $S_{v}$ are the intraspecies interaction strengths and $C$ is the interspecies interaction strength.

\section{Numerical Method}

We now derive the numerical scheme for equation (1) in one dimension,

$$
\frac{\partial u}{\partial t}=\frac{\partial}{\partial x}\left(\mathbf{v}_{u} u\right)+D \frac{\partial^{2} u}{\partial x^{2}}
$$

where

$$
\mathbf{v}_{u}=-\frac{\partial}{\partial x}\left(Q_{u} * \max \left\{\left(S_{u} u+C v\right)(1-u-v), 0\right\}\right) .
$$

The numerical scheme for (2) is derived in a similar manner and thus omitted here. For the numerical scheme, the terms described in Table 1 are used in regards to an arbitrary grid point $i$.

We begin by discretising the diffusion term using a standard 4th order central differencing scheme. Using the terms in Table 1, we get

$$
\frac{\partial^{2} u}{\partial x^{2}} \approx \frac{D}{12 \Delta x^{2}}\left(-U_{i+2}+16 U_{i+1}-30 U_{i}+16 U_{i-1}-U_{i-2}\right) .
$$

In order to solve the velocity component of equation 1 we exploit the convolution theorem, which states that under suitable conditions the Fourier transform of a convolution of two functions is equal to the point-wise product of their individual Fourier transforms, i.e.,

$$
\mathscr{F}\{f * g\}=\mathscr{F}\{f\} \cdot \mathscr{F}\{g\}
$$



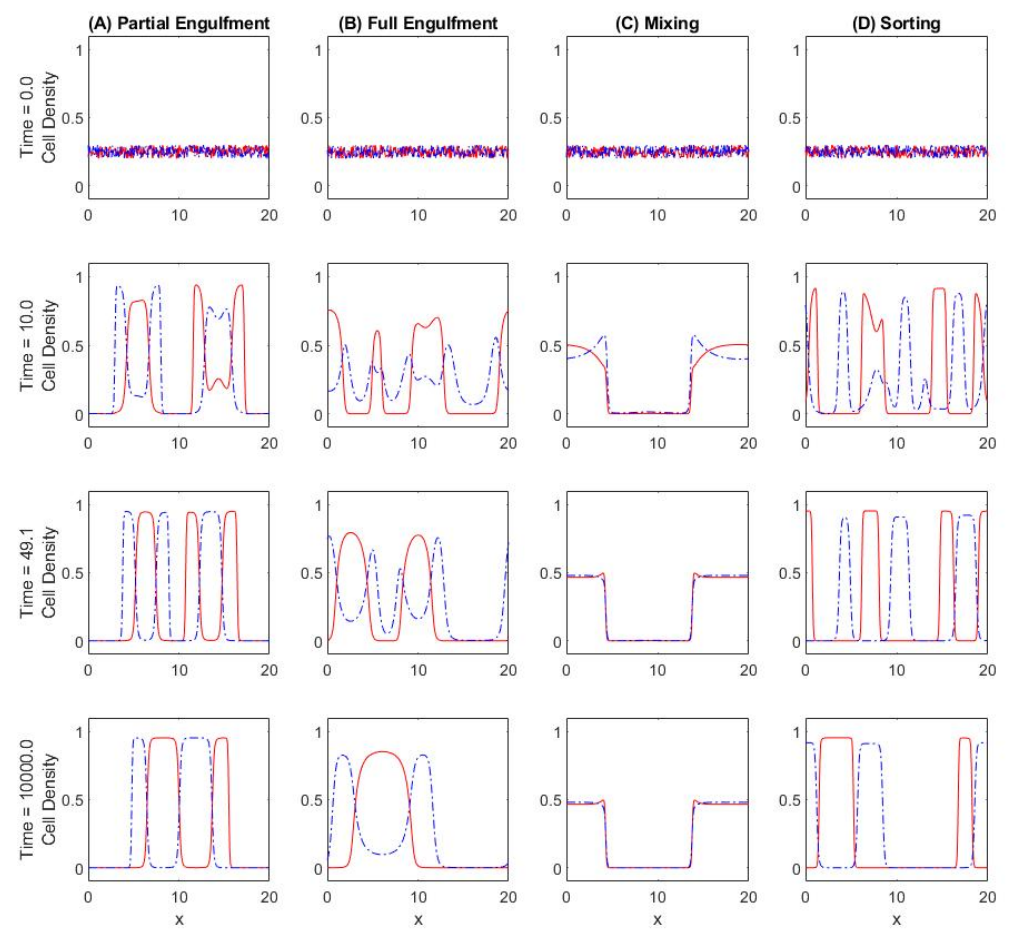

Figure 1. Numerical simulation results using adhesive strengths related to Armstrong et al. (2006). The spatial domain is a length of 20 divided up with 256 gird points. Results are displayed at $t=0,10$, and 49.1 with a steady state at $t=10000$. The results use the following adhesion values. (A) $S_{u}=25, S_{v}=25, C=12.5$, (B) $S_{u}=25, S_{v}=2.5, C=5$, (C) $S_{u}=25, S_{v}=7.5, C=22.5$, (D) $S_{u}=25, S_{v}=7.5, C=0$, with $D=0.1$ and $r=1$ in all simulations. The results give excellent agreement with Armstrong et al. (2006).

Where $\mathscr{F}$ represents the Fourier transform (we will also denote the inverse Fourier transform as $\mathscr{F}^{-1}$ ). Additionally, we use the following property of convolutions

$$
\frac{\partial}{\partial x}(f * g)=\left(\left(\frac{\partial}{\partial x} f\right) * g\right)=\left(f *\left(\frac{\partial}{\partial x} g\right)\right),
$$

to turn the velocity component of the advection term into

$$
\mathbf{v}_{u}=\mathscr{F}^{-1}\left\{\mathscr{F}\left\{-\frac{\partial}{\partial x} Q_{u}\right\} \cdot \mathscr{F}\left\{\max \left\{\left(S_{u} u+C v\right)(1-u-v), 0\right\}\right\}\right\} .
$$

As it is very computationally efficient, we use Fast Fourier Transforms (FFTs). However, this requires a periodic domain and to properly implement the convolution we have to perform some modifications to our social interaction functions $Q_{u, v}$. We require $Q_{u, v}(x)$ to be periodic on the domain $x \in[0, L]$, thus we write

$$
-\frac{\partial}{\partial x} Q_{u}= \begin{cases}0 & \text { if } x=0, \\ \frac{1}{r}\left(e^{-\frac{|x|}{r}}-e^{\frac{|x-(L+\Delta x)|}{r}}\right) & \text { if } x \neq 0 .\end{cases}
$$

We can then calculate the velocity function as

$$
\mathbf{v}_{u} \approx \Delta x \cdot \text { real }\left\{\operatorname{ifft}\left\{\operatorname{fft}\left\{-\frac{\partial}{\partial x} Q_{u}(\mathbf{x})\right\} \cdot \operatorname{fft}\left\{\max \left\{\left(S_{u} \mathbf{U}+C \mathbf{V}\right)(1-\mathbf{U}-\mathbf{V}), 0\right\}\right\}\right\}\right\},
$$

where fft and ifft represent the fast Fourier transform and inverse fast Fourier transform respectively. We take only the real component of the ifft as any imaginary value will be simply due to error. We then calculate the 


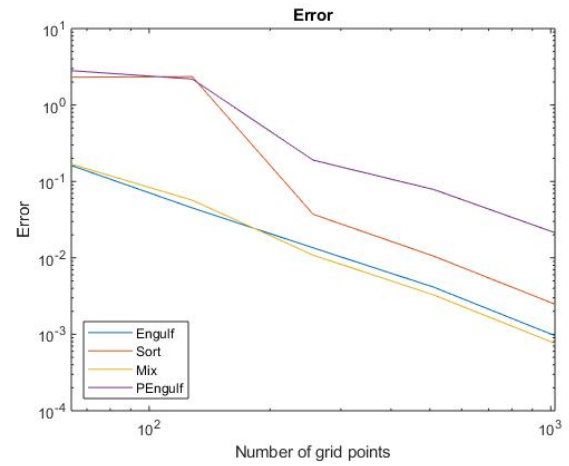

Figure 2. Numerical error estimates at $t=10000$ for engulfment (Engulf), mixing (Mix), sorting (Sort), and partial engulfment (PEngulf). We used 2048 spatial points a reference solution.

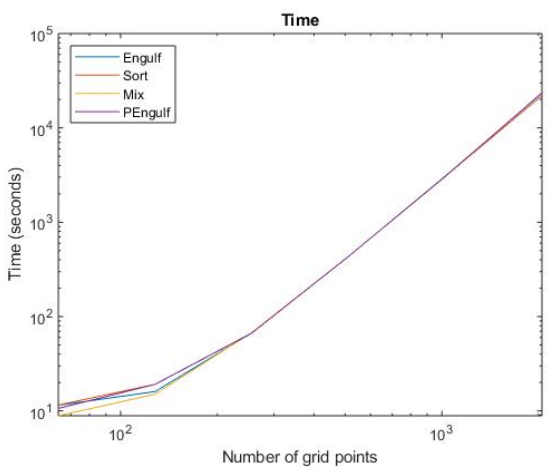

Figure 3. Computation time for $t=10000$ for engulfment (Engulf), mixing (Mix), sorting (Sort), and partial engulfment (PEngulf). All calculations were done on a single CPU with 16GB of ram.

advection term using a fourth order central differencing scheme

$$
\frac{\partial}{\partial x}\left(\mathbf{v}_{u} u\right) \approx \frac{1}{12 \Delta x}\left(-v_{u, i+2} U_{i+2}+8 v_{u, i+1} U_{i+1}-8 v_{u, i-1} 6 U_{i-1}+v_{u, i-2} U_{i-2}\right) .
$$

Finally, for time integration we use the Dormand-Prince method (Dormand and Prince (1980)). This is an adaptive Runge-Kutta method where the coefficients are chosen in such a way that it is possible to calculate a 4th and 5th order scheme simultaneously. The 5th order scheme is then used as an error estimate for the 4th order scheme. This error estimate is then used to adapt the time step (see Loan (1997)).

\section{Results}

In order to verify our our model, we compare our results against those of Armstrong et al. (2006). We run our simulations with periodic boundary conditions, i.e., $u(t, L)=u(t, 0)$. The spatial domain is $x=[0,20]$, the time domain is $t=[0,10000]$, and initial conditions are $u(0, x)=0.2+\mu, v(0, x)=0.2+\mu$ where $\mu$ is some uniformly distributed noise, $\mu \sim \mathcal{U}(0,0.1)$. To implement our numerical scheme we divide the spatial domain up into 256 grid points, this allows us to use FFTs to solve the convolution at each time step.

The results are shown in Figure 1. We obtained the same behaviours as that of Armstrong et al. (2006). However, the results tend towards a single cellular mass as opposed to disjoint aggregations.

The error can be seen in Figure 2. In order to calculate this, we set 2048 spatial points as our reference solution (denoted $\mathbf{U}_{r e f}$ and $\mathbf{V}_{r e f}$ ), then for each number of grid points $(64,128,256,512$, and 1024) we calculate our total error, $\epsilon_{\text {total }}$, as

$$
\epsilon_{\text {total }}=\left\|\mathbf{U}_{r e f}-\mathbf{U}\right\|+\left\|\mathbf{V}_{\text {ref }}-\mathbf{V}\right\|
$$


where $\|\cdot\|$ represents the standard $L_{2}$ norm. Error is calculated at the pseudo steady state of $t=10000$. From this we can estimate the convergence rate as greater than 1.7 for each type of cell behaviour $(1.8448,2.4665$, 1.9465, 1.7603 for engulfment, sorting, mixing, and partial engulfment respectively).

The main advantage of this method is the reduction in computation time, when comparing to previous simulations of Armstrong et al. (2006). In particular from the numerical technique adopted in Georgiou et al. (2019) we are able to obtain a 1000 times decrease in computation time compared to a fixed grid and a 10 times decrease when compared to our prior gradient based refinement method for 1024 grid points. Finally, the computation time for $t=10000$ can be seen in Figure 3

\section{ACKNOWLedgement}

The authors are grateful to the University of Newcastle for the provision of a PhD scholarship for F.G.

\section{REFERENCES}

Armstrong, N. J., K. J. Painter, and J. A. Sherratt (2006, Nov). A continuum approach to modelling cell-cell adhesion. Journal of theoretical biology 243(1), 98-113.

Bernoff, A. and C. Topaz (2013, Jan). Nonlocal aggregation models: A primer of swarm equilibria. SIAM Review 55(4), 709-747.

Dormand, J. R. and P. J. Prince (1980, Mar). A family of embedded runge-kutta formulae. Journal of Computational and Applied Mathematics 6(1), 19-26.

Foty, R. A. and M. S. Steinberg (2005, Feb). The differential adhesion hypothesis: a direct evaluation. Developmental Biology 278(1), 255-263.

Georgiou, F., B. P. Lamichhane, and N. Thamwattana (2019, October). An adaptive numerical scheme for a partial integro-differential equation. In B. Lamichhane, T. Tran, and J. Bunder (Eds.), Proceedings of the 18th Biennial Computational Techniques and Applications Conference, CTAC-2018, Volume 60 of ANZIAM J., pp. C187-C200. http: / / journal.austms.org.au/ojs/index.php/ANZIAMJ / article/view/14066 [October 9, 2019].

Loan, C. F. V. (1997). Introduction to Scientific Computing: A Matrix Vector Approach Using MATLAB.

Loan, O. J. and M. R. Evans (1999, Jan). Alternating steady state in one-dimensional flocking. Journal of Physics A: Mathematical and General 32(8), L99-L105.

Mogilner, A. and L. Edelstein-Keshet (1999, Jun). A non-local model for a swarm. Journal of Mathematical Biology 38(6), 534-570.

Sheratt, J. A., S. A. Gourley, N. J. Armstrong, and K. J. Painter (2009). Boundedness of solutions of a nonlocal reaction-diffusion model for adhesion in cell aggregation and cancer invasion. European Journal of Applied Mathematics 20(1), 123-144.

Steinberg, M. S. (1962a). Mechanism of tissue reconstruction by dissociated cells, ii: Time-course of events. Science 137(3532), 762-763.

Steinberg, M. S. (1962b). On the mechanism of tissue reconstruction by dissociated cells, i. population kinetics, differential adhesiveness and the absence of directed migration. Proceedings of the National Academy of Sciences of the United States of America 48(9), 1577-1582.

Steinberg, M. S. (1962c). On the mechanism of tissue reconstruction by dissociated cells, iii. free energy relations and the reorganisation of fused, heteronomic tissue fragments. Proceedings of the National Academy of Sciences of the United States of America 48(10), 1769-1776.

Topaz, C. M., M. R. D’Orsogna, L. Edelstein-Keshet, and A. J. Bernoff (2012, Aug). Locust dynamics: Behavioral phase change and swarming. PLOS Computational Biology 8(8), e1002642. 